\title{
Protective effects of paeonol on subacute/chronic brain injury during cerebral ischemia in rats
}

\author{
BING ZHAO $^{1 *}$, QIAO-JUAN SHI ${ }^{1,2^{*}}$, ZHEN-ZHONG ZHANG ${ }^{3}$, SHU-YAN WANG ${ }^{1}$, XI WANG $^{4}$ and HAO WANG $^{3}$ \\ ${ }^{1}$ Department of Anesthesiology, The First Affiliated Hospital, School of Medicine, \\ Zhejiang University; ${ }^{2}$ Experimental Animal Center, Zhejiang Academy of Medical Sciences, Hangzhou, \\ Zhejiang 310013; ${ }^{3}$ Department of Neurology, Tongde Hospital of Zhejiang Province, Hangzhou, Zhejiang 310012; \\ ${ }^{4}$ Key Laboratory of Digestive Pathophysiology of Zhejiang Province, Zhejiang Hospital of Traditional Chinese Medicine, \\ Zhejiang Chinese Medical University, Hangzhou, Zhejiang 310006, P.R. China
}

Received May 10, 2017; Accepted October 5, 2017

DOI: $10.3892 /$ etm.2018.5893

\begin{abstract}
Ischemic stroke is a highly complex pathological process that is divided into acute, subacute and chronic phases. Paeonol is a biologically active natural product with a variety of pharmacological effects, including those on neuronal activity. However, the effects of paeonol on subacute/chronic ischemic stroke have remained to be elucidated. The present study was designed to investigate the effects of paeonol against subacute and chronic cerebral ischemic injury and to explore the possible underlying mechanisms. Male adult Sprague Dawley rats were randomly divided into a sham group (treated with saline), a model group [subjected to middle cerebral artery occlusion (MCAO) and treated with saline] and a paeonol-treated group ( $\mathrm{MCAO}+$ paeonol at $25 \mathrm{mg} / \mathrm{kg}$ ). Behavioral impairment, infarct volume and ischemic/contralateral hemispheric ratios were assessed at $72 \mathrm{~h}$ and at 28 days after MCAO, respectively. Immunofluorescence was employed to determine the neuronal damage and glial responses after MCAO. Compared with the model group, paeonol treatment significantly attenuated behavioral impairment, ischemic infarct volume and moderate cerebral edema in the ischemic brain at $72 \mathrm{~h}$, as well as brain atrophy at 28 days after reperfusion. Furthermore, paeonol treatment ameliorated neuronal damage in the ischemic core and boundary zone regions at $72 \mathrm{~h}$ after reperfusion and in the boundary zone at 28 days after reperfusion. In addition, paeonol treatment reduced the proliferation of astrocytes in the boundary zone, and inhibited microglial activation in the ischemic core and boundary
\end{abstract}

Correspondence to: Dr Hao Wang, Department of Neurology, Tongde Hospital of Zhejiang Province, 234 Gucui Road, Hangzhou, Zhejiang 310012, P.R. China

E-mail: wangh8234@163.com

*Contributed equally

Key words: paeonol, subacute/chronic brain injury, cerebral ischemia, rat zone regions at $72 \mathrm{~h}$ and 28 days after reperfusion. These results demonstrated the protective effects of paeonol against subacute/chronic cerebral ischemia, and the mechanism of action may include subacute/chronic microglial activation and astrocyte proliferation.

\section{Introduction}

Ischemic stroke is the primary cause of cerebrovascular diseases and remains to be one of the leading causes of death and disability in patients worldwide $(1,2)$. Despite tremendous research efforts leading to improvements in ischemic stroke treatment, the available therapeutic strategies are currently limited, and several promising agents evaluated in extensive preclinical trials failed to enter clinical trials $(3,4)$. At present, thrombolytic therapy remains to be the gold standard for ischemic stroke treatment; however, it is only efficacious within a narrow therapeutic window $(1,2,4,5)$. Hence, beyond the therapeutic window, management of stroke mainly depends on supportive therapy, secondary prevention and rehabilitation (1). Thus, an enhanced understanding regarding the pathological processes may provide novel therapeutic options to promote stroke recovery.

Ischemic stroke is a highly complex pathological process, which induces a series of cellular and molecular events and may be divided into three stages: The acute phase (hours), the subacute phase (hours to days) and the chronic phase (days to months) (6). In the acute stage, metabolic disturbances and excitotoxicity are involved in the progression of neuronal damage; in the subacute period, inflammation and cell death are the dominant events; in the chronic phase, brain repair is the major response and includes microgliosis, glial scar formation, angiogenesis, as well as neuronal regeneration (6-8). In addition, during the pathological process of ischemic stroke, the subacute and chronic phases are generally termed as late (subacute/chronic) phases $(6,9)$. In the late phase, one of the dominant responses is reactive astrogliosis with subsequent glial scar formation, which not only protects the neurons against harmful substances by isolating the injury area but also obstructs neuronal regeneration by suppressing axonal sprouting $(10,11)$. Another important event is the activation 
of microglia, which endangers neuronal survival by releasing numerous proinflammatory and neurotoxic mediators $(8,12,13)$.

Recently, considerable attention has been paid to Traditional Chinese Medicine, as it includes sources of neuroprotective components (14). Natural products, particularly medicinal plants, may provide an ideal choice for the development of safe and effective drugs for stroke treatment (14). Paeonol, an active component isolated from the Chinese herbal medicine Cortex Moutan, which is the root bark of Paeonia suffruticosa Andr., has been demonstrated to possess diverse pharmacological activities, including anti-oxidant (15), anti-atherosclerotic (16), anti-tumor (17), anti-diabetic (18), and anti-inflammatory effects (19). Regarding its applicability for central nervous system diseases, previous studies have demonstrated that paeonol exerts neuroprotective actions against acute ischemic stroke, as well as Parkinson's and Alzheimer's disease in animal models, (19-22). These studies indicate that paeonol may be a promising drug for the treatment of neurological disorders. However, to the best of our knowledge, the potential effects of paeonol on subacute/chronic ischemic stroke have remained to be determined. The present study pursued to investigate the therapeutic potential of paeonol in middle cerebral artery occlusion (MCAO)-induced subacute/chronic cerebral ischemia. Furthermore, the present study focused on the post-ischemic microglial and astrocyte responses in the rat brain in an attempt to elucidate the underlying mechanisms.

\section{Materials and methods}

Experimental animals. A total of 125 adult male Sprague Dawley rats weighing 230-280 g (10-12 weeks old) were obtained from the Animal Science Center of Zhejiang Academy of Medical Sciences, [Hangzhou, China; certificate no. SCXK (Zhe) 2014-0001]. Animals were housed in an animal center at a constant temperature of $22 \pm 2^{\circ} \mathrm{C}$, a relative humidity of $50 \pm 10 \%$ and a 12 -h light/dark cycle. They were allowed free access to food and water. Behavioral experiments were arranged at 10:00 a.m.-05:00 p.m. during the day. All efforts were made to minimize their suffering. All experimental procedures were performed in accordance with the National Institutes of Health Guidelines for the Care and Use of Experimental Animals and were approved by the Animal Care and Use Committee of Zhejiang University (Hangzhou, China).

Induction of transient focal cerebral ischemia. Transient focal cerebral ischemia was induced by MCAO according to previous methods (23) with certain modifications, such as using the nylon suture with a poly-L-lysine coated and without performing the ligation of the pterygopalatine artery $(9,24)$. In brief, after animals were anesthetized by intraperitoneal (i.p.) injection chloral hydrate $(400 \mathrm{mg} / \mathrm{kg})$, and the left common carotid artery, the left external carotid artery (ECA) and the left internal carotid artery (ICA) were isolated. A nylon suture coated with poly-L-lysine was inserted into the ICA from the ECA until a slight resistance was felt, which indicated blockage of the MCA origin. Occlusion was performed for a period of $30 \mathrm{~min}$, after which the suture was removed to allow for reperfusion. After the surgery, the animals were placed in a warm box and allowed to recover from anesthesia. The sham animals underwent the same surgical procedures without insertion of the suture. During the experiment, the change in regional cerebral blood flow ( $\mathrm{rCBF}$ ) was monitored as described previously with the steady-state baseline prior to the operation being regarded as $100 \%(9,25)$. In addition, the blood pressure, $\mathrm{PaO}_{2}, \mathrm{PaCO}_{2}$, glucose and $\mathrm{pH}$ were continuously monitored as described previously during the entire course of the operation $(9,25)$. At the designated endpoint, all animals were euthanized by inhalation of $\mathrm{CO}_{2}$, and additional animals were allocated to ensure that a sufficient number of animals survived until the designated endpoint. Animals exhibiting signs including subarachnoid hemorrhage, or a moribund or comatose state with labored respiration, were excluded and euthanized with $\mathrm{CO}_{2}(26,27)$.

Drug administration and groups. Paeonol was obtained from Sigma-Aldrich (Merck KGaA, Darmstadt, Germany) with a purity of $>99 \%$ and dissolved in saline at a concentration of $2 \mathrm{mg} / \mathrm{ml}$. Based on a preliminary experiment, a dose of $25 \mathrm{mg} / \mathrm{kg}$ paeonol (i.p.) was used in the present study, which exerted the best neuroprotective effect in acute cerebral ischemia [2,3,5-triphenyltetrazolium chloride (TTC) staining and histopathological results; data not shown]. The regimen of drug administration applied in the present study was established in previous studies $(9,24)$.

To evaluate the effects of paeonol on subacute ischemic injury, the animals were randomly divided into a sham group (saline, $\mathrm{n}=16$ ), a model group (MCAO + saline, $\mathrm{n}=23$ ) and a paeonol-treated group (MCAO + Pae at $25 \mathrm{mg} / \mathrm{kg}, \mathrm{n}=21$ ). For drug delivery, the same volume of saline or paeonol solution was injected i.p. at the onset of MCAO (when the nylon suture was inserted and the MCA origin was blocked), and then injected once a day for 3 days. During the experiment, one part of the animals $(n=8)$ were used for determination of infarct volume, and the remaining animals were prepared to cut frozen sections for immunostaining. The $10-\mu \mathrm{m}$ frozen sections at 2-mm intervals from the frontal to the occipital poles were cut by cryomicrotomy (CM1900, Leica, Wezlar, Germany).

To evaluate the effects of paeonol on chronic ischemic injury, the animals were randomly divided into a sham group (saline, $\mathrm{n}=16)$, a model group ( $\mathrm{MCAO}+$ saline, $\mathrm{n}=26)$ and a paeonol-treated group ( $\mathrm{MCAO}+\mathrm{Pae}$ at $25 \mathrm{mg} / \mathrm{kg}, \mathrm{n}=23$ ) in another separate experiment. For drug delivery, the same volume of saline or paeonol was injected i.p. at the onset of $\mathrm{MCAO}$, then injected once a day from days 2-7, and then once every 2 days from days 8-28.

Behavioral assessment. The neurological deficit score was determined at the indicated time-points according to the established scoring system: In the absence of neurological deficits, the score of 0 was given, upon failure to extend right paw fully, the score of 1 was given, animals circling to the right received the score of 2 , falling to the right was scored as 3 , and no spontaneous walking and depressed levels of consciousness was scored as 4 (23). The test was repeated for three times and the average value was recorded.

The inclined board test was performed to evaluate the balance and coordination of animals according to previous research (28) with certain modifications, including the 
material and size of the board, and the rotation angle (24). Animals were placed on the board $(50 \times 30 \mathrm{~cm})$, and once they were stable, the board was inclined from horizontal to vertical at a rate of $2 \%$ sec. The holding angle was defined as the angle at which the animal fell off the board. The test was repeated for three times and the average value was calculated.

One observer who was blinded to the experimental groups performed all of the assessments.

Determination of infarct volume. After the neurological assessment, one part of the animals was re-anesthetized, and the brains were quickly removed and coronally sliced into 6 sections of $2 \mathrm{~mm}$ in thickness. The slices were then incubated in $0.5 \%$ 2,3,5-triphenyltetrazolium chloride (TTC) at $37^{\circ} \mathrm{C}$ for $20 \mathrm{~min}$, followed by fixation in $4 \%$ paraformaldehyde for $2 \mathrm{~h}$. Subsequently, images of the fixed slices were captured. The infarction area was identified as the unstained area in the brain sections. The infarct volume was calculated by summing up the volumes of the 6 slices, and represented as the percentage of infarction in the total brain hemisphere.

Histological analysis. Following the behavioral tests, the remaining animals were re-anesthetized and then transcardially perfused with $4 \%$ paraformaldehyde after a pre-flush with saline. The brains were quickly removed, and were immediately immersed in $4 \%$ paraformaldehyde for $24 \mathrm{~h}$, followed by dehydration in $30 \%$ sucrose for 3 days, then images were captured with a digital camera. Finally, a series of sections $(10 \mu \mathrm{m})$ from the frontal to the occipital poles were cut by cryomicrotomy (CM1900; Leica Microsystems, Wetzlar, Germany). The sections were prepared for immunostaining.

To determine the changes in the number of different cell types, immunofluorescence staining was performed. The $10-\mu \mathrm{m}$ slides were rinsed with PBS and then incubated with $10 \%$ normal goat serum (Zhongshan Belling Biotechnology Co., Ltd., Beijing, China) for $2 \mathrm{~h}$ at room temperature. Subsequently, the sections were incubated at $4^{\circ} \mathrm{C}$ overnight with the following primary antibodies: Mouse anti-glial fibrillary acidic protein (GFAP; cat. no. MAB3402; 1:800 dilution; EMD Millipore, Billerica, MA, USA), mouse anti-neuronal nuclei (NeuN; cat. no. MAB377; 1:200 dilution; EMD Millipore) and rabbit anti-ionized calcium binding adaptor molecule 1 (Iba1; cat. no. 019-19741; 1:1,000 dilution; Wako Pure Chemical Industries, Ltd., Wako, Japan). Thereafter, sections were washed three times in PBS and incubated with fluorescein isothiocyanate-conjugated secondary antibody (1:200 diluton; cat. nos. AP132F or AP124F; EMD Millipore) for $2 \mathrm{~h}$ at room temperature. For the negative control, PBS was applied instead of the primary antibodies. Images of the stained sections were captured using a fluorescence microscope (Olympus BX51: Olympus, Tokyo, Japan). Eight non-overlapping images for each site of the same rats were randomly selected, and the average value was determined. The neurons and the microglia were calculated according to the average number of stained cells and the astrocytes were calculated according to the average fluorescence intensity.

Statistical analysis. Values are expressed as the mean \pm standard error of the mean. Significance of differences was assessed by one-way analysis of variance, followed by
Dunnett's post-hoc test (SPSS 10.0 for Windows; SPSS, Inc., Chicago, IL, USA). The results of the behavioral assessments were analyzed using the Kruskal-Wallis test. $\mathrm{P}<0.05$ was considered to indicate a statistically significant difference.

\section{Results}

Physiological changes after the operation. No significant differences in the physiological parameters, including mean arterial blood pressure, partial pressure of $\mathrm{CO}_{2}\left(\mathrm{PaCO}_{2}\right), \mathrm{PaO}_{2}$, blood glucose, $\mathrm{pH}$ and weight were identified between $30 \mathrm{~min}$ prior to and after the operation. After cerebral ischemia, the $\mathrm{rCBF}$ was $\sim 70 \%$ decreased at $30 \mathrm{~min}$ after occlusion, and then returned to baseline levels after reperfusion $(\mathrm{P}<0.01$; Table I). Paeonol did not affect these physiological variables, including the reduction of $\mathrm{rCBF}$ (Table I). During the 28 days, all of the sham animals were survived. However, no significant difference in the survival rate was identified between the model group (61.54\%) and the paeonol-treated group (69.57\%), and these rates were consistent with those reported by previous studies (29).

Effect of paeonol on behavioral impairment. In the subacute experiment, behavioral impairments were evaluated at 24 (data not shown) and $72 \mathrm{~h}$ after reperfusion (Fig. 1). It was demonstrated that the neurological deficit scores and the holding angle were aggravated at $72 \mathrm{~h}$ after reperfusion. Compared with those in the sham group, the neurological deficit scores were significantly increased in the MCAO rats, whereas the holding angle in the inclined board test was significantly decreased $(\mathrm{P}<0.01$; Fig. 1A and $\mathrm{C})$. By contrast, a significant alleviation in the behavioral impairment was observed in the paeonol-treated group compared with that in the model group $(\mathrm{P}<0.05$; Fig. 1A and $\mathrm{C})$.

In the chronic experiment, the measures of behavioral impairment, including the neurological deficit score and the holding angle, were aggravated at $72 \mathrm{~h}$ after reperfusion, and then gradually recovered at 7,14 and 28 days after reperfusion (data not shown). Compared with the model group, paeonol administration led to a more efficient improvement in neurological scores and the holding angle in rats at $72 \mathrm{~h}$ after reperfusion. However, no significant difference in the behavioral impairments was identified between these groups from days 28 after reperfusion due to the chronic recovery $(\mathrm{P}>0.05$; Fig. 1B and D).

Effect of paeonol on infarct volumes and ischemic/contralateral hemispheric ratio. The representative images of total brains and the TTC-stained coronal slices indicated swelling/atrophy on the surface and infarctions in the hemispheres after cerebral ischemia, respectively (Fig. 2A-B).

The effects of paeonol on subacute brain injury at $72 \mathrm{~h}$ after reperfusion were assessed. Compared with those in the sham group, the infarct volume and the ischemic/contralateral hemispheric ratio, an index of brain edema, were significantly increased at $72 \mathrm{~h}$ after reperfusion $(\mathrm{P}<0.01$; Fig. $1 \mathrm{E}$ and $\mathrm{G}$, respectively, and Fig. 2A). However, compared with that in the model group, paeonol treatment significantly alleviated the increase of infarct volume and ischemic/contralateral hemispheric ratio at $72 \mathrm{~h}$ after reperfusion $(\mathrm{P}<0.05$ or $\mathrm{P}<0.01$; 
Table I. Physiological variables prior to and after operation.

\begin{tabular}{|c|c|c|c|}
\hline \multirow[b]{2}{*}{ Variable } & \multirow[b]{2}{*}{ Sham } & \multicolumn{2}{|c|}{ Ischemia } \\
\hline & & Model & $\mathrm{Pae}$ \\
\hline Body weight (g) & $262.3 \pm 11.4$ & $272.8 \pm 14.3$ & $264.4 \pm 11.6$ \\
\hline \multicolumn{4}{|l|}{ MABP (mmHg) } \\
\hline Baseline & $105.2 \pm 10.1$ & $102.3 \pm 8.9$ & $110.0 \pm 14.7$ \\
\hline 30 min after reperfusion & $111.8 \pm 11.2$ & $110.1 \pm 14.3$ & $118.5 \pm 10.2$ \\
\hline \multicolumn{4}{|l|}{$\mathrm{Pa}_{\mathrm{O} 2}(\mathrm{mmHg})$} \\
\hline Baseline & $109.2 \pm 4.1$ & $105.2 \pm 4.5$ & $103.8 \pm 5.3$ \\
\hline 30 min after reperfusion & $104.7 \pm 3.0$ & $99.8 \pm 4.1$ & $101.2 \pm 5.6$ \\
\hline \multicolumn{4}{|l|}{$\mathrm{Pa}_{\mathrm{CO} 2}(\mathrm{mmHg})$} \\
\hline Baseline & $38.3 \pm 5.1$ & $40.4 \pm 6.2$ & $39.4 \pm 4.1$ \\
\hline 30 min after reperfusion & $41.2 \pm 3.9$ & $42.8 \pm 4.3$ & $44.3 \pm 4.9$ \\
\hline \multicolumn{4}{|l|}{$\mathrm{pH}$} \\
\hline Baseline & $7.45 \pm 0.38$ & $7.38 \pm 0.41$ & $7.39 \pm 0.43$ \\
\hline 30 min after reperfusion & $7.40 \pm 0.42$ & $7.48 \pm 0.39$ & $7.46 \pm 0.36$ \\
\hline \multicolumn{4}{|l|}{ Glucose (g/l) } \\
\hline Baseline & $6.58 \pm 0.81$ & $6.85 \pm 0.64$ & $6.12 \pm 0.53$ \\
\hline 30 min after reperfusion & $7.10 \pm 0.75$ & $6.99 \pm 0.68$ & $7.08 \pm 0.65$ \\
\hline \multicolumn{4}{|l|}{$\mathrm{rCBF}(\%)$} \\
\hline Baseline & 100 & 100 & 100 \\
\hline 30 min after ischemia & $98.5 \pm 6.2$ & $35.8 \pm 7.5^{\mathrm{a}}$ & $34.6 \pm 5.7^{\mathrm{a}}$ \\
\hline 30 min after reperfusion & $98.9 \pm 5.8$ & $93.6 \pm 8.3$ & $95.1 \pm 7.9$ \\
\hline
\end{tabular}

Fig. 1E and G, respectively, and Fig.2A). Furthermore, the effects of paeonol on chronic brain injury were assessed at 28 days after reperfusion. Compared with that in the sham group, the ischemic/contralateral hemispheric ratio was markedly decreased at 28 days after reperfusion $(\mathrm{P}<0.01$; Figs. $1 \mathrm{H}$ and $2 \mathrm{~B})$, indicating brain atrophy, which was consistent with the results of previous studies $(9,29,30)$. By contrast, administration of paeonol markedly alleviated chronic brain atrophy in comparison with that in the model group $(\mathrm{P}<0.05$; Figs. $1 \mathrm{H}$ and $2 \mathrm{~B})$. However, no significant differences were identified in the infarct volumes between the paeonol-treated and model groups ( $\mathrm{P}>0.05$; Figs. $1 \mathrm{~F}$ and $2 \mathrm{~B}$ ). This may have been due to the apparent atrophy, which hindered the observation of further changes, and the chronic functional recovery. Collectively, these results indicated that paeonol exerted neuroprotective effects on subacute and chronic cerebral ischemic injury.

Effect of paeonol on neuronal damage. To assess the effect of paeonol on ischemic neuronal injury, the changes of neuronal damage in the ischemic brains after paeonol treatment were observed (Fig. 3). Cerebral ischemia induced evident ischemic lesions exhibiting neuronal injury, which included the shrinkage of cell bodies that were deeply stained using Nisssl staining, and the disappearance of Nissl bodies (data not shown). In the ischemic core, compared with that in the sham group, the density of neurons identified by immunofluorescent staining for NeuN was markedly decreased at $72 \mathrm{~h}$ after reperfusion, and disappeared at 28 days after reperfusion $(\mathrm{P}<0.01$; Fig. 3A, $\mathrm{B}$ and $\mathrm{D})$. In the boundary zone, neuronal density was markedly decreased at $72 \mathrm{~h}$ and 28 days after reperfusion $(\mathrm{P}<0.01$; Fig. 3A, C and E). Paeonol treatment significantly ameliorated neuronal loss in the ischemic core, as well as in the boundary zone at $72 \mathrm{~h}$ after reperfusion in comparison with that in the model group $(\mathrm{P}<0.01$; Fig. 3A-C). In addition, paeonol treatment markedly inhibited neuronal loss in the boundary zone at 28 days after reperfusion ( $\mathrm{P}<0.01$; Fig. $3 \mathrm{~A}$ and $\mathrm{E})$. However, no significant differences in the neuronal density in the ischemic core area were observed between the paeonol-treated and model groups, as neurons disappeared at 28 days after reperfusion in the ischemic core area (Fig. 3A and D). However, a limited number of degenerated neurons or background staining may be observed (Fig. 3A and D).

Effect of paeonol on astrocyte proliferation. To determine the underlying mechanisms involved in the neuroprotective effects of paeonol on subacute and chronic ischemic injury, the present study further assessed the astrocyte responses in the ischemic hemisphere after paeonol administration (Fig. 4). The results indicated that the density of GFAP-positive astrocytes was not significantly changed at $24 \mathrm{~h}$ after 

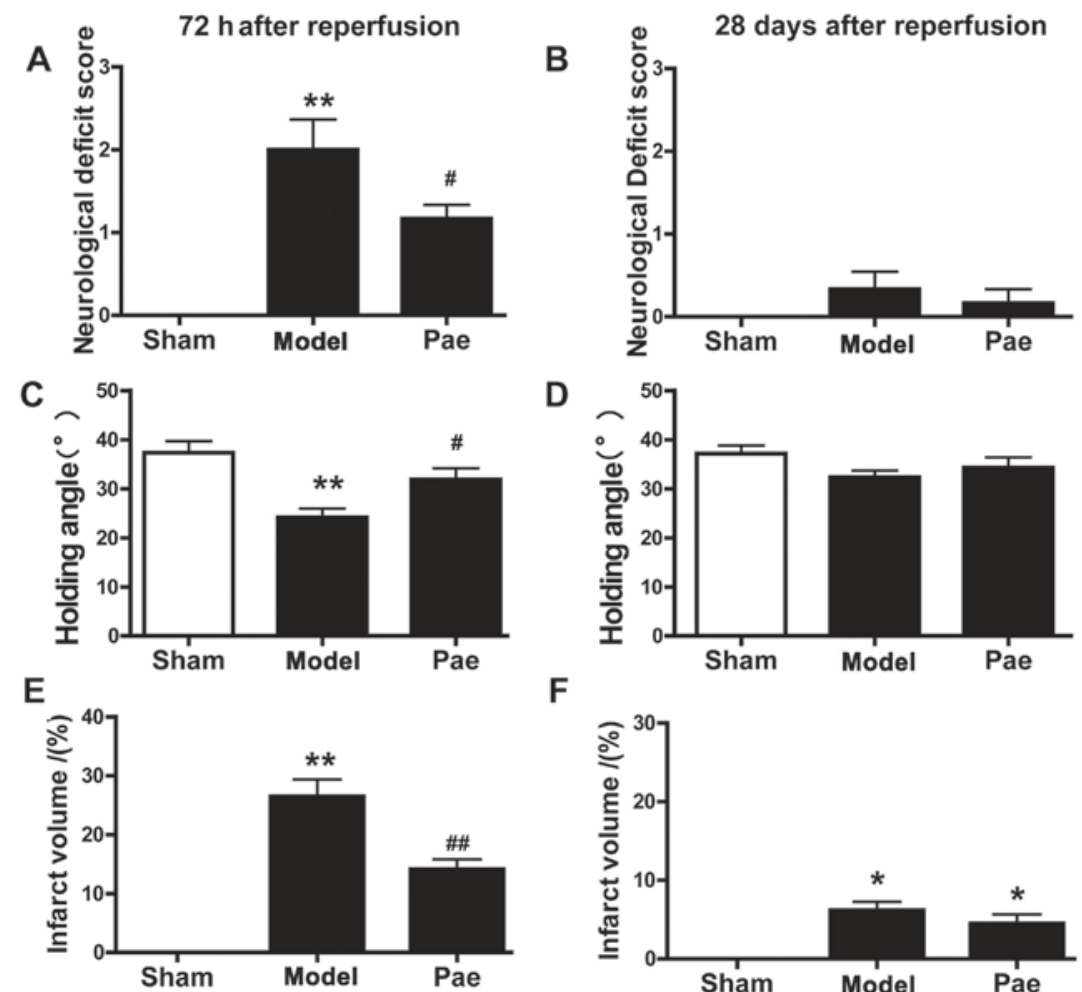

F
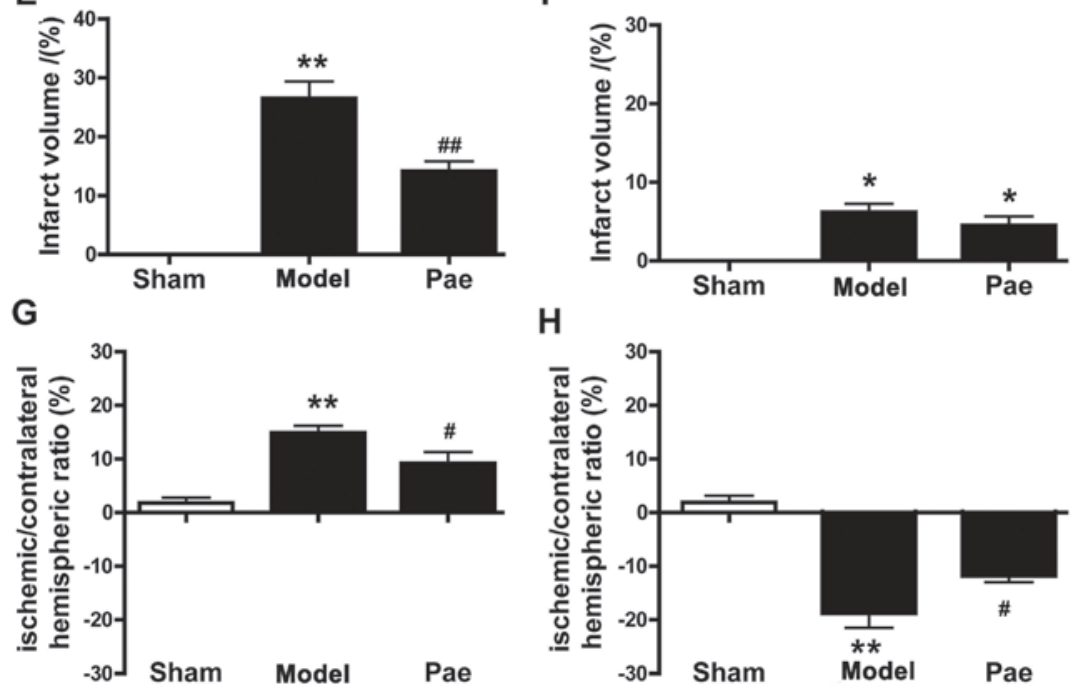

Figure 1. Effects of paeonol on neurological dysfunction, infarct volume and the ischemic/contralateral hemispheric ratio after focal cerebral ischemia. (A-D) Paeonol treatment significantly ameliorated the neurological deficit score and holding angle at $72 \mathrm{~h}$ after reperfusion compared with those in the model group; however, it did not affect the already recovered neurological deficit score and holding angle at 28 days after reperfusion. (E and F) Paeonol treatment significantly decreased the infarct volume at $72 \mathrm{~h}$ after reperfusion compared with that in the model group; however, it did not affect the already decreased infarct volume at 28 days after reperfusion. ( $\mathrm{G}$ and $\mathrm{H}$ ) The ischemic/contralateral hemispheric ratio was increased (brain edema) at $72 \mathrm{~h}$ and decreased (brain atrophy) at 28 days after reperfusion, and these changes were significantly ameliorated by paeonol treatment. Values are expressed as the mean \pm standard error of the mean from eight rats per group. ${ }^{*} \mathrm{P}<0.05$ and ${ }^{* * *} \mathrm{P}<0.01$ compared with sham group, ${ }^{\prime \prime} \mathrm{P}<0.05$ and ${ }^{\# \#} \mathrm{P}<0.01$ compared with model group. Pae, paeonol.

reperfusion (data not shown). However, at $72 \mathrm{~h}$ after reperfusion, astrocytes in the boundary zone were significantly increased and hypertrophied, and a glial scar surrounding the ischemic core area had formed at 28 days after reperfusion $(\mathrm{P}<0.01$; Fig. 4A, C and E). By contrast, in the ischemic core area, astrocytes were initially decreased at $72 \mathrm{~h}$ after reperfusion, and eventually disappeared at 28 days after reperfusion (Fig. 4A, B and D). Paeonol treatment significantly reduced the density of GFAP-positive astrocytes in the boundary zone at $72 \mathrm{~h}$ and 28 days after reperfusion compared with that in the model group $(\mathrm{P}<0.01$; Fig. $4 \mathrm{~A}, \mathrm{C}$ and $\mathrm{E})$. However, no significant differences were observed in the density of GFAP-positive astrocytes in the ischemic core area between the paeonol-treated and model group at $72 \mathrm{~h}$ and 28 days after reperfusion, and the astrocytes gradually disappeared from this area (Fig. 4A, B and D). Overall, the results indicated that paeonol exerted its beneficial effects by reducing post-ischemic astrocyte proliferation.
Effect of paeonol on microglial activation. To evaluate whether microglial activation was associated with the neuroprotective effects of paeonol on post-ischemic injury, immunostaining analysis for microglia was performed. Microglia are inflammatory cells that are rapidly activated after brain injury. Activation of microglia involves their proliferation, migration into the injured area, upregulation of various immunomodulators and phagocytosis of the damaged cells and debris. In the cerebral cortex of sham rats, ramified Iba-1-positive microglial cells were diffusely distributed (Fig. 5A). At $24 \mathrm{~h}$ after reperfusion, the number of Iba-1-positive microglia was not particularly changed, but the of Iba-1-positive microglia morphology became irregular (data not shown). However, the number of Iba-1-positive cells, including that of the ramified microglia and activated (ameboid/round) microglia/macrophages, was gradually increased at $72 \mathrm{~h}$ after reperfusion compared with that in the sham group $(\mathrm{P}<0.01$; Fig. 5A-C). In addition, at 28 days after 

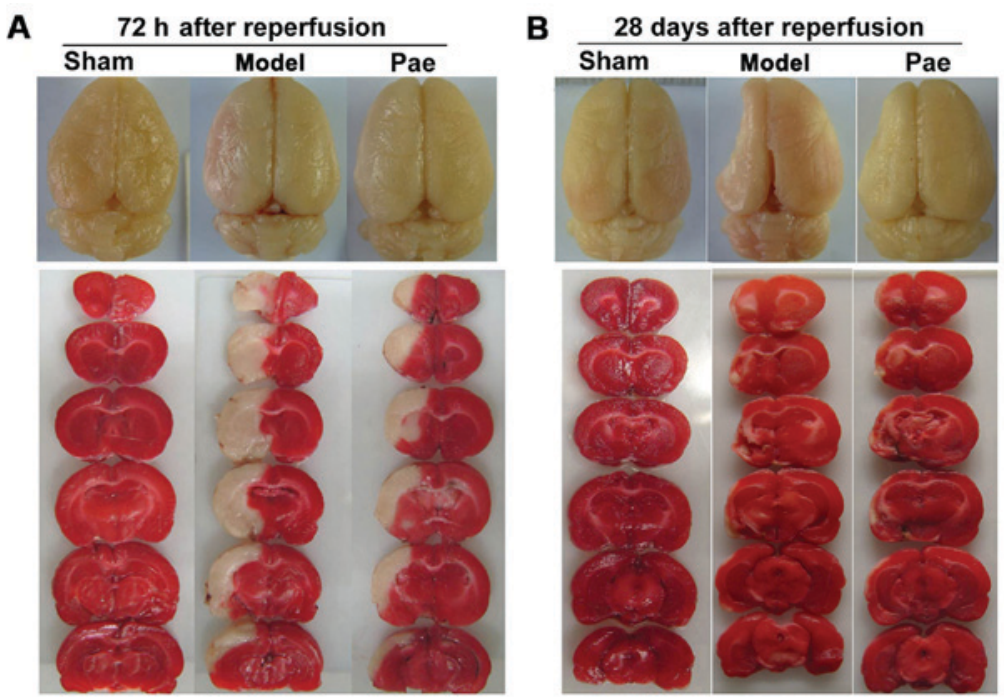

Figure 2. Effects of paeonol on ischemic brain injury. Representative images of total brains indicated brain lesions at (A) $72 \mathrm{~h}$ and (B) 28 days after reperfusion (upper panel). Representative images of TTC-stained coronal slices with $2 \mathrm{~mm}$-thickness indicated ischemic lesions at (A) $72 \mathrm{~h}$ and (B) 28 days after reperfusion (lower panel). After TTC staining, the viable tissue stained deep red, whereas the infarct area was white in color. TTC, 2,3,5-triphenyltetrazolium chloride; Pae, paeonol.

reperfusion, the number of Iba-1-positive cells, particularly that of activated (ameboid/round) microglia/macrophages, was markedly increased in the ischemic core and boundary zone regions compared with that in the sham group $(\mathrm{P}<0.01$; Fig. 5A, D and E). By contrast, compared with that in the model group, paeonol treatment significantly inhibited the increase in Iba-1-positive microglia and ameliorated the morphological changes in the ischemic core, as well as the boundary zone at $72 \mathrm{~h}$ and 28 days after reperfusion $(\mathrm{P}<0.01$; Fig. 5A-E). These results indicated the protective effects of paeonol, which were associated with post-ischemic microglial activation.

\section{Discussion}

Traditional Chinese Medicine has been increasingly recognized and has demonstrated a therapeutic significance in the treatment of ischemic stroke (14). Paeonol, an active phenolic component of Cortex Moutan, has been demonstrated to possess diverse biological properties (16,17,19-22). Paeonol has been proved to be effective in treating acute experimental ischemic stroke by inhibiting the excitotoxicity, calcium overload and oxidative stress $(19,20)$. However, to date, the effects of paeonol against subacute/chronic cerebral ischemic injury have remained elusive. Therefore, the present study investigated the effects of paeonol on subacute/chronic cerebral ischemic injury and further determined the underlying mechanisms.

The amphiphilic structure and low molecular weight of paeonol facilitate the easy penetration of the blood brain barrier (19). Thus, in the present study, paeonol was administered to rats by intraperitoneal injection, and its effect against subacute/chronic cerebral ischemic injury was explored. Numerous animal studies on ischemia have established a model where the injury at $24 \mathrm{~h}$ following ischemia/reperfusion is regarded as acute injury of cerebral ischemia, that at $72 \mathrm{~h}$ to 7 days after reperfusion is regarded as subacute injury and that at 14-35 days following ischemia/reperfusion is regarded as chronic injury $(6,9,29,31)$. Thus, in the present study, the ischemic injury in rats was observed at $72 \mathrm{~h}$ and 28 days after cerebral ischemia/reperfusion. The behavioral impairments in these rats were also evaluated in $24 \mathrm{~h}$ after reperfusion (data not shown). Consistent with the previous studies, the behavioral impairments were aggravated at $24 \mathrm{~h}$ after reperfusion, and paeonol treatment improved their neurological deficits. The major results of the present study demonstrated that the increase in the infarct volume and ischemic/contralateral hemispheric ratio (edema) in the ischemic hemisphere at $72 \mathrm{~h}$ after reperfusion and the decrease in the ischemic/contralateral hemispheric ratio (atrophy) at 28 days after reperfusion were significantly attenuated by paeonol treatment. Furthermore, paeonol treatment greatly ameliorated the behavioral impairment at $72 \mathrm{~h}$ after reperfusion compared with that in the model group. In addition, paeonol treatment significantly ameliorated neuronal damage in the ischemic core and the boundary zone regions at $72 \mathrm{~h}$ after reperfusion, and in the boundary zone at 28 days after reperfusion. Taken together, it was indicated that paeonol had protective effects on subacute and chronic cerebral ischemic injury.

In addition to the above changes, the present study further investigated the potential mechanisms underlying the protective effects of paeonol on subacute and chronic ischemic stroke. As is known, astrocytes and microglia have pivotal roles in the progression of ischemic stroke $(10,32)$. Accumulating evidence suggests that glial responses require hours to days to fully develop, and may in turn provide potential targets for stroke recovery with longer therapeutic windows compared with those of other treatments $(3,13)$. In addition, post-ischemic inflammation is a crucial step during the process of ischemic stroke $(13,33)$. Thus, the present study subsequently focused on the effects of paeonol on inflammation-associated glial cells.

During the post-ischemic phase, one important event in subacute/chronic ischemic brain injury is reactive astrogliosis and glial scar formation $(10,11)$. As a vital part of the neurovascular unit, astrocytes have an important role in the 
A MCAO
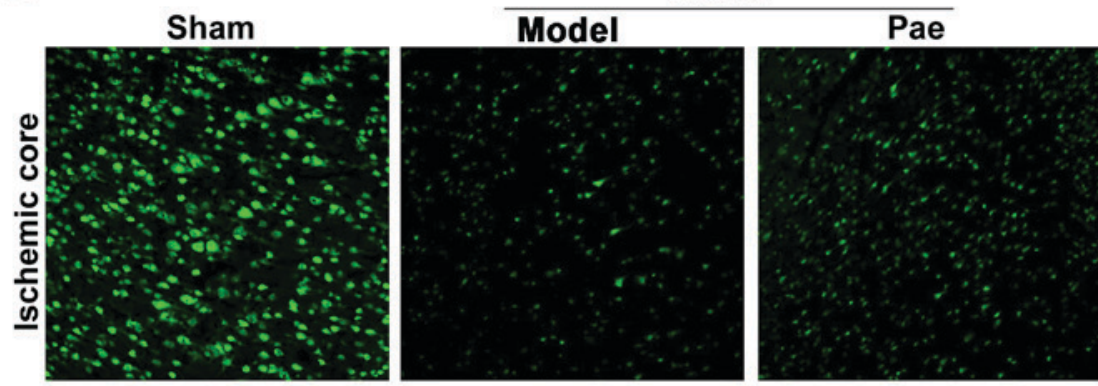

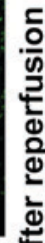
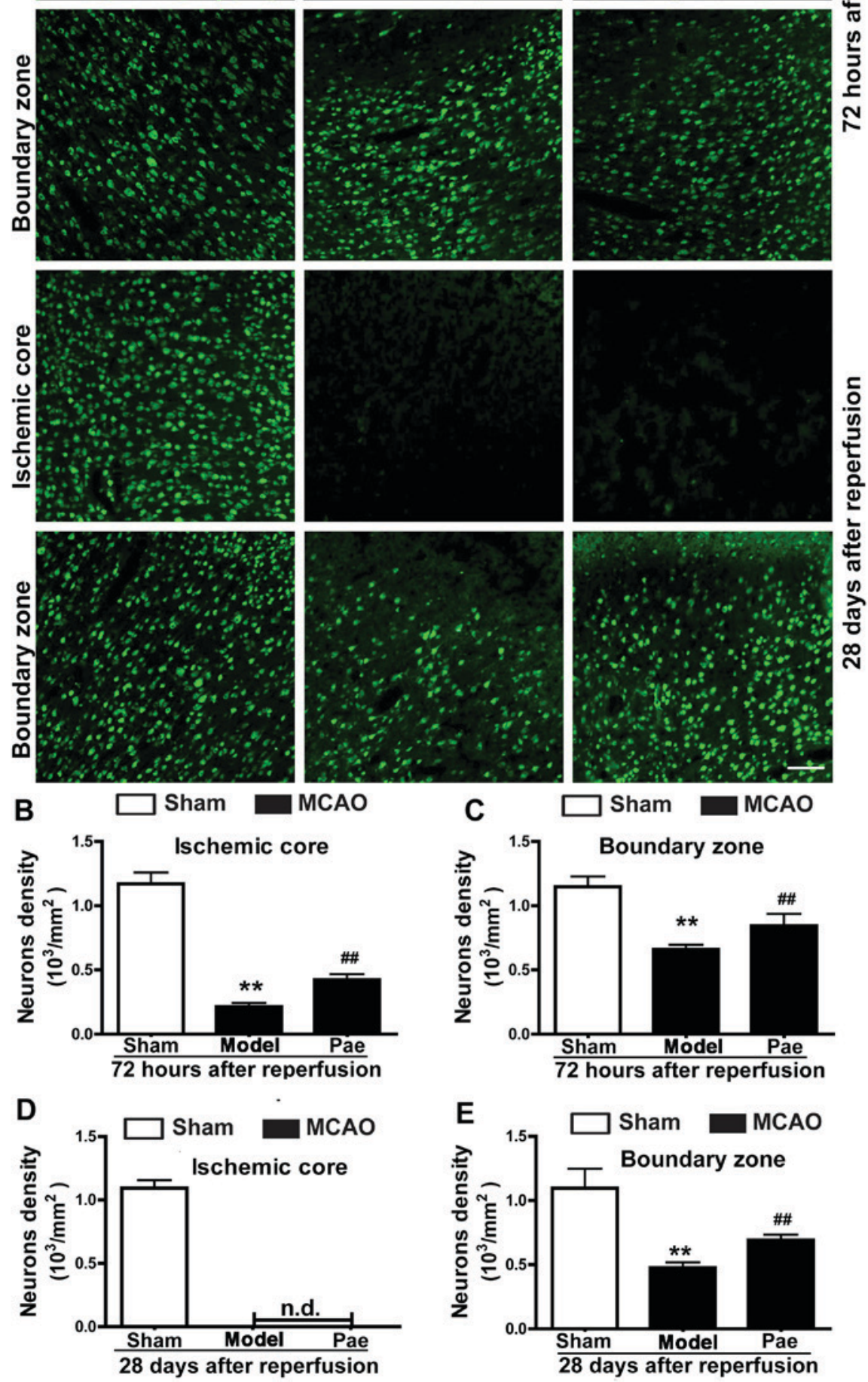

Figure 3. Effects of paeonol on neuronal injury at $72 \mathrm{~h}$ and 28 days after reperfusion in rats. (A) Representative photomicrographs indicated neurons by immunostaining for NeuN at $72 \mathrm{~h}$ and at 28 days after reperfusion (scale bar, $100 \mu \mathrm{m}$ ) and (B-E) quantification results. In the ischemic core, the reduction of neuronal density was significantly attenuated by paeonol treatment at $72 \mathrm{~h}$ after reperfusion, whereas NeuN-expressing cells completely disappeared at 28 days after reperfusion. In the boundary zone, the decrease of neuronal density was significantly attenuated by paeonol treatment at $72 \mathrm{~h}$ and 28 days after reperfusion. Values are expressed as the mean \pm standard error of the mean from eight rats per group. ${ }^{* *} \mathrm{P}<0.01$ compared with sham group, ${ }^{\# \#} \mathrm{P}<0.01$ compared with model group. Pae, paeonol; MCAO, middle cerebral artery occlusion; n.d., not detectable; NeuN, neuronal nuclei. 

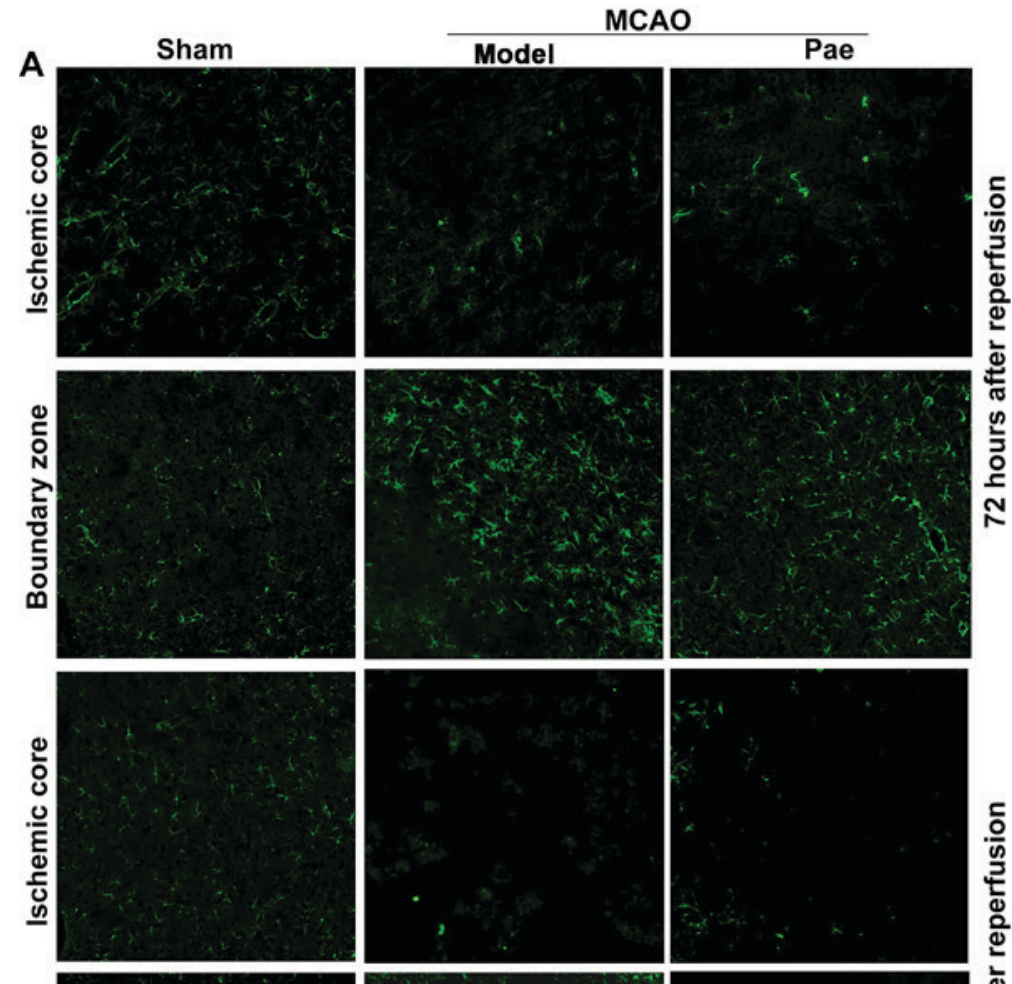

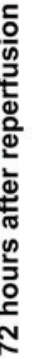
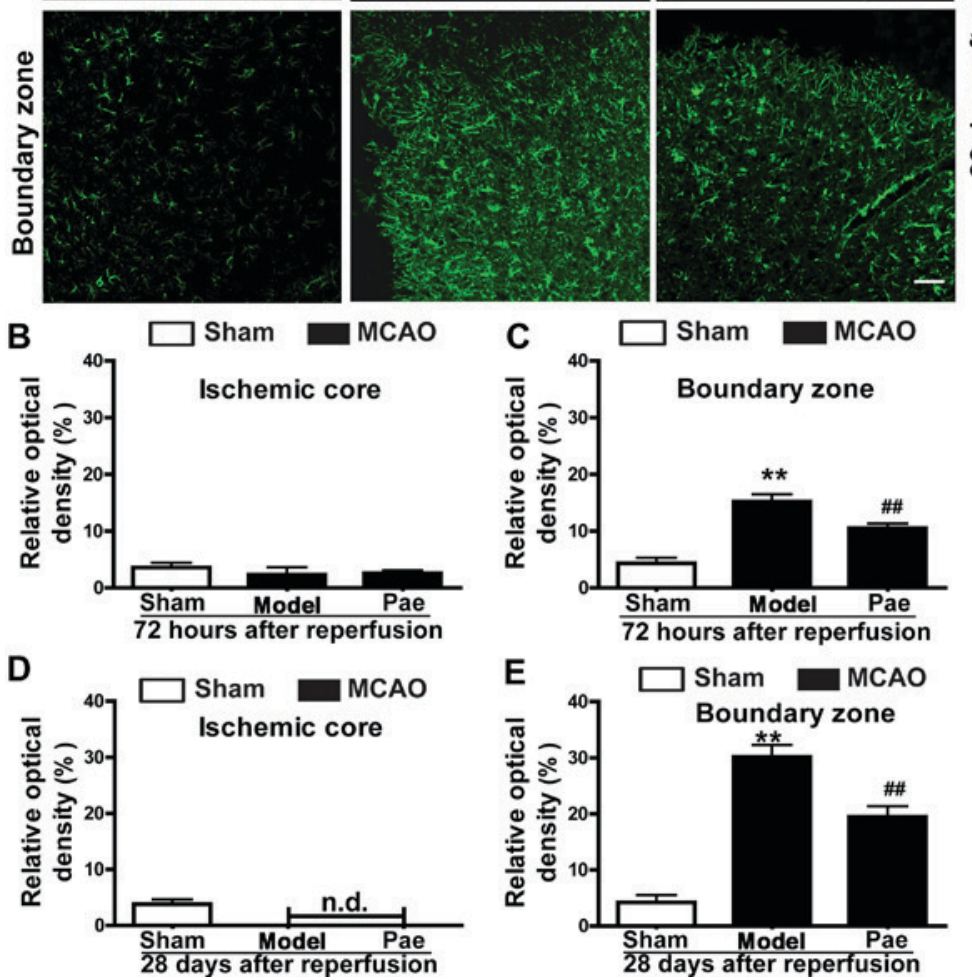

Figure 4. Effects of paeonol on astrocyte proliferation at $72 \mathrm{~h}$ and 28 days after reperfusion in rats. (A) Representative photomicrographs indicating glial fibrillary acidic protein-immunopositive astrocytes at $72 \mathrm{~h}$ and 28 days after reperfusion (scale bar, $100 \mu \mathrm{m}$ ) and (B-E) quantification results. At $72 \mathrm{~h}$ after reperfusion, astrocytes were initially increased in the boundary zone, and paeonol treatment significantly suppressed the changes in the boundary zone, but not those in the ischemic core. At 28 days after reperfusion, astrocytes were significantly increased in the boundary zone, and paeonol treatment significantly attenuated the astrogliosis in the boundary zone, but not that in the ischemic core. Values are expressed as the mean \pm standard error of the mean from eight rats per group. ${ }^{* *} \mathrm{P}<0.01$ compared with sham group, ${ }^{\# \#} \mathrm{P}<0.01$ compared with model group. Pae, paeonol; $\mathrm{MCAO}$, middle cerebral artery occlusion; n.d., not detectable.

physiology of the normal brain, which includes blood circulation, extracellular ionic homeostasis, release of energy substrates and growth factors, as well as neurotransmission $(3,11)$. Numerous studies have demonstrated that astrocytes protect the neurons from glutamate excitotoxicity during ischemic stroke $(10,11)$. However, increasing evidence has indicated that astrocytes paradoxically exacerbate ischemic injury with morphological and phenotypic changes; this process is termed reactive gliosis or astrogliosis $(7,10)$. Following focal ischemic injury, reactive astrocytes migrate towards the boundary zone 

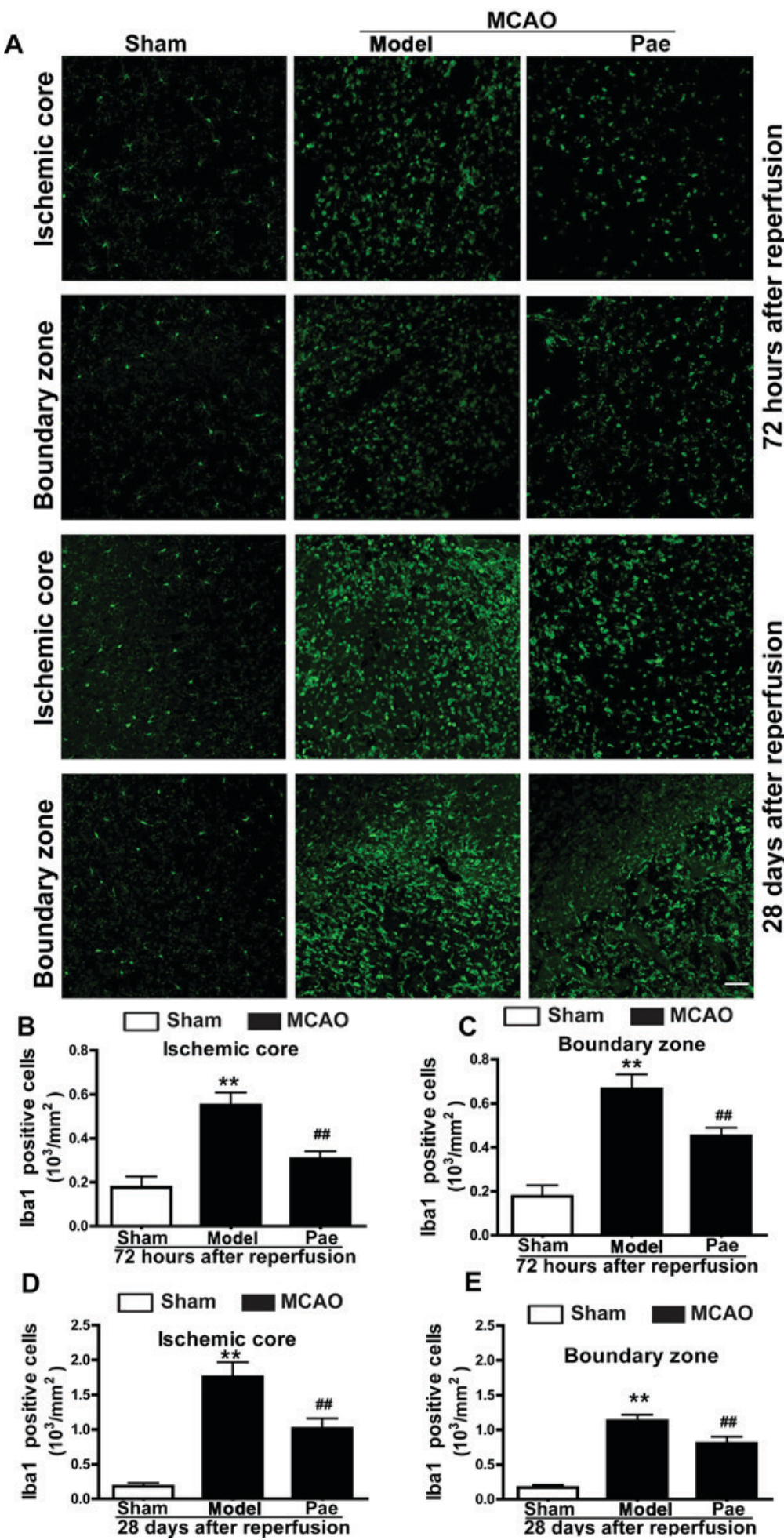

Figure 5. Effects of paeonol on microglial activation at $72 \mathrm{~h}$ and 28 days after reperfusion in rats. (A) Representative photomicrographs displaying Iba-1-immunopositive microglia in the ischemic core and boundary zone regions at $72 \mathrm{~h}$ and 28 days after reperfusion (scale bar, $100 \mu \mathrm{m}$ ) and (B-E) quantification results. At $72 \mathrm{~h}$ after reperfusion, Iba-1-positive microglia initially increased in the ischemic core and the boundary zone regions, and these changes were significantly inhibited by paeonol treatment. At 28 days after reperfusion, microglial cells in the ischemic core and boundary zone regions were significantly increased, which was attenuated by paeonol treatment. Values are expressed as the mean \pm standard error of the mean from eight rats per group. ${ }^{* *} \mathrm{P}<0.01$ compared with sham group, ${ }^{\# \#} \mathrm{P}<0.01$ compared with model group. Pae, paeonol; MCAO, middle cerebral artery occlusion; Iba- 1 , ionized calcium binding adaptor molecule 1 .

and then eventually organize into the glial scar that separates the ischemic core region from healthy tissue $(3,7,10)$. The formation of a glial scar is a critical event in the brain repair responses after ischemic injury, which act as a physical and biochemical barrier that separates the viable and dead tissues, but also obstructs neuronal regeneration by suppressing axonal sprouting $(3,10,11)$. Growing evidence suggests that modulation of reactive astrocytes may be a promising strategy for the 
management of stroke (11). In the present study, paeonol treatment significantly reduced GFAP-positive astrocytes in the boundary zone at $72 \mathrm{~h}$ and 28 days after reperfusion compared with those in the model group, but not in the ischemic core, which may be due to the gradual disappearance of astrocytes in the area. Different from the mechanisms observed during acute cerebral ischemic injury, paeonol treatment may regulate the astrocyte responses and inhibit undesired outcomes, including glial scar formation, because of its association with the obstruction neuronal regeneration by suppressing axonal sprouting. Thus, the present study provided valuable details regarding the role of paeonol in modulating post-ischemic astrocyte proliferation.

With regard to the changes in the late phase, another important event in post-ischemic brain injury is microgliosis and the associated inflammatory responses $(13,32,34)$. Microglial cells are the resident immune cells and are the first and major line of active immune defense in the intact or injured brain $(12,13)$. During neuropathological conditions, microglial cells are rapidly activated to restore homeostasis of the central nervous system $(12,35)$. The activation of microglia involves their proliferation and migration into the injured area, upregulation of various immunomodulators, phagocytose of the damaged cell debris and antigenic substances $(8,33,34)$. However, microglial activation has dual effects, and uncontrolled or overactivated microglia are detrimental for the pathological conditions (35). Activated microglia may aggravate neuronal damage through the release of nitric oxide, reactive oxygen species, protease and proinflammatory cytokines such as tumor necrosis factor- $\alpha$, as well as interleukin-1 $\beta$ and $-6(35,36)$. Chronic microglial activation has been considered to be the possible underlying mechanism in the neuronal damage and is associated with numerous neurodegenerative diseases, including ischemic stroke $(12,13)$. Therefore, appropriate identification of agents that inhibit microglial activation may act as effective treatment strategies for providing neuroprotection $(12,35)$. In the present study, paeonol treatment significantly inhibited the increase in Iba-1-positive microglia and ameliorated the morphological changes in the ischemic core and boundary zone areas at $72 \mathrm{~h}$ and 28 days after reperfusion. Among the various biological properties, the anti-inflammatory activities of paeonol have been demonstrated in macrophages, microglia and in the BV2 cell line in vitro, which was consistent with the present in vivo results $(37,38)$. It was indicated that paeonol treatment may regulate microglial activation, suggesting the potential use of paeonol in the treatment of post-ischemic microgliosis.

In summary, the present study indicated the protective effects of paeonol and its involvement in the treatment of subacute/chronic cerebral ischemia, particularly in the late phase of microglial activation and astrocyte proliferation. During subacute cerebral ischemia, paeonol effectively alleviated neurological impairment, reduced the infarct volume, cerebral edema and neuronal loss, and suppressed microglial activation and astrocyte proliferation. During chronic cerebral ischemia, paeonol markedly ameliorated brain atrophy and neuronal loss, and inhibited microglial proliferation and astrocyte proliferation. Based on the above results obtained in rats, understanding the long-term neuroprotective effects of paeonol in the process of chronic cerebral ischemia may lead to the development of a novel clinical treatment for ischemic stroke. Overall, the present study broadened the current knowledge on the range of pharmacological properties of paeonol for the treatment of ischemic stroke. However, the precise molecular mechanisms of the neuroprotective effects of paeonol require elucidation in future studies.

\section{Acknowledgements}

Not applicable.

\section{Funding}

The present study was supported by grants from the National Natural Science Foundation (grant nos. 81401566, 31301933, 81301122 and 81400594), the Zhejiang Provincial Natural Science Foundation (grant no. LQ15H090005) and the Science and Technology Planning Project of Zhejiang Province (grant nos. 2015KYB076 and 2015ZB012).

\section{Availability of data and materials}

The datasets used and/or analyzed during the current study are available from the corresponding author on reasonable request.

\section{Authors' contributions}

BZ, QJS and HW designed the research. BZ, QJS, ZZZ, HW performed the experiments and data collection. BZ, SYW and XW analyzed the data. BZ, QJS and HW wrote the manuscript. All authors have read and approved this version of the article, and ensure the integrity of the work.

\section{Ethics approval and consent to participate}

All experimental procedures were performed in accordance with the National Institutes of Health Guidelines for the Care and Use of Experimental Animals and were approved by the Animal Care and Use Committee of Zhejiang University (Hangzhou, China).

\section{Consent for publication}

Not applicable.

\section{Competing interests}

The authors declare that they have no competing interests.

\section{References}

1. Hankey GJ: Stroke. Lancet 389: 641-654, 2017.

2. Jauch EC, Saver JL, Adams HP Jr, Bruno A, Connors JJ, Demaerschalk BM, Khatri P, McMullan PW Jr, Qureshi AI, Rosenfield K, et al: Guidelines for the early management of patients with acute ischemic stroke: A guideline for healthcare professionals from the American Heart Association/American Stroke Association. Stroke 44: 870-947, 2013.

3. Li L and Stary CM: Targeting glial mitochondrial function for protection from cerebral ischemia: Relevance, mechanisms, and the role of MicroRNAs. Oxid Med Cell Longev 2016: 6032306, 2016. 
4. Hacke W, Kaste M, Bluhmki E, Brozman M, Dávalos A, Guidetti D, Larrue V, Lees KR, Medeghri Z, Machnig T, et al: Thrombolysis with alteplase 3 to $4.5 \mathrm{~h}$ after acute ischemic stroke. N Engl J Med 359: 1317-1329, 2008.

5. Shichita T, Ago T, Kamouchi M, Kitazono T, Yoshimura A and Ooboshi $\mathrm{H}$ : Novel therapeutic strategies targeting innate immune responses and early inflammation after stroke. J Neurochem 123 (Suppl 2): S29-S38, 2012

6. Fagan SC, Hess DC, Hohnadel EJ, Pollock DM and Ergul A: Targets for vascular protection after acute ischemic stroke. Stroke 35: 2220-2225, 2004.

7. Burda JE and Sofroniew MV: Reactive gliosis and the multicellular response to CNS damage and disease. Neuron 81: 229-248, 2014.

8. Hanisch UK and Kettenmann H: Microglia: Active sensor and versatile effector cells in the normal and pathologic brain. Nat Neurosci 10: 1387-1394, 2007.

9. Zhao B, Zhao CZ, Zhang XY, Huang XQ, Shi WZ, Fang SH, Lu YB, Zhang WP, Xia Q and Wei EQ: The new P2Y-like receptor $\mathrm{G}$ protein-coupled receptor 17 mediates acute neuronal injury and late microgliosis after focal cerebral ischemia in rats. Neuroscience 202: 42-57, 2012

10. Choudhury GR and Ding S: Reactive astrocytes and therapeutic potential in focal ischemic stroke. Neurobiol Dis 85: 234-244, 2016.

11. Liu Z and Chopp M: Astrocytes, therapeutic targets for neuroprotection and neurorestoration in ischemic stroke. Prog Neurobiol 144: 103-120, 2016.

12. Kim JY, Kim N and Yenari MA: Mechanisms and potential therapeutic applications of microglial activation after brain injury. CNS Neurosci Ther 21: 309-319, 2015.

13. Jin $X$ and Yamashita T: Microglia in central nervous system repair after injury. J Biochem 159: 491-496, 2016.

14. Bu Y, Lee K, Jung HS and Moon SK: Therapeutic effects of traditional herbal medicine on cerebral ischemia: A perspective of vascular protection. Chin J Integr Med 19: 804-814, 2013.

15. Ding Y, Li Q, Xu Y, Chen Y, Deng Y, Zhi F and Qian K Attenuating oxidative stress by paeonol protected against Acetaminophen-induced hepatotoxicity in mice. PLoS One 11: e0154375, 2016.

16. Liu YR, Chen JJ and Dai M: Paeonol protects rat vascular endothelial cells from ox-LDL-induced injury in vitro via downregulating microRNA-21 expression and TNF- $\alpha$ release. Acta Pharmacol Sin 35: 483-488, 2014

17. Li M, Tan SY and Wang XF: Paeonol exerts an anticancer effect on human colorectal cancer cells through inhibition of PGE, synthesis and COX-2 expression. Oncol Rep 32: 2845-2853, 2014.

18. Liu J, Feng L, Ma D, Zhang M, Gu J, Wang S, Fu Q, Song Y, Lan Z, Qu R and Ma S: Neuroprotective effect of paeonol on cognition deficits of diabetic encephalopathy in streptozotocin-induced diabetic rat. Neurosci Lett 549: 63-68, 2013.

19. Liao WY, Tsai TH, Ho TY, Lin YW, Cheng CY and Hsieh CL: Neuroprotective effect of paeonol mediates anti-inflammation via suppressing Toll-like receptor 2 and Toll-like receptor 4 signaling pathways in cerebral ischemia-reperfusion injured rats. Evid Based Complement Alternat Med 2016: 3704647, 2016.

20. Zhao Y, Fu B, Zhang X, Zhao T, Chen L, Zhang J and Wang X: Paeonol pretreatment attenuates cerebral ischemic injury via upregulating expression of pAkt, Nrf2, HO-1 and ameliorating BBB permeability in mice. Brain Res Bull 109: 61-67, 2014.

21. Shi X, Chen YH, Liu H and Qu HD: Therapeutic effects of paeonol on methyl-4-phenyl-1,2,3,6-tetrahydropyridine/probenecid-induced Parkinson's disease in mice. Mol Med Rep 14: 2397-2404, 2016.
22. Zhou A, Wu H, Pan J, Wang X, Li J, Wu Z and Hui A: Synthesis and evaluation of paeonol derivatives as potential multifunctional agents for the treatment of Alzheimer's disease. Molecules 20 : $1304-1318,2015$

23. Longa EZ, Weinstein PR, Carlson S and Cummins R: Reversible middle cerebral artery occlusion without craniectomy in rats. Stroke 20: 84-91, 1989.

24. Shi QJ, Wang H, Liu ZX, Fang SH, Song XM, Lu YB, Zhang WP, Sa XY, Ying HZ and Wei EQ: HAMI 3379, a CysLT2R antagonist, dose- and time-dependently attenuates brain injury and inhibits microglial inflammation after focal cerebral ischemia in rats. Neuroscience 291: 53-69, 2015.

25. Yano T, Anraku S, Nakayama R and Ushijima K: Neuroprotective effect of urinary trypsin inhibitor against focal cerebral ischemia-reperfusion injury in rats. Anesthesiology 98: 465-473, 2003

26. Graham SM, McCullough LD and Murphy SJ: Animal models of ischemic stroke: Balancing experimental aims and animal care Comp Med 54: 486-496, 2004

27. Rewell SS, Churilov L, Sidon TK, Aleksoska E, Cox SF, Macleod MR and Howells DW: Evolution of ischemic damage and behavioural deficit over 6 months after MCAo in the rat: Selecting the optimal outcomes and statistical power for multi-centre preclinical trials. PLoS One 12: e0171688, 2017.

28. Yonemori F, Yamaguchi T, Yamada H and Tamura A: Evaluation of a motor deficit after chronic focal cerebral ischemia in rats. J Cereb Blood Flow Metab 18: 1099-1106, 1998.

29. Lu LQ, Lou Q, Guo HG, Zhou WW, Ying HZ, Wei EQ and Shi QJ: Neuroprotective effect of HAMI 3379, a CysLT2R receptor antagonist, on chronic brain injury after focal cerebral ischemia in rats. Int J Clin Exp Pathol 10: 4123-4136, 2017.

30. Shen LH, Li Y, Chen J, Cui Y, Zhang C, Kapke A, Lu M, Savant-Bhonsale S and Chopp M: One-year follow-up after bone marrow stromal cell treatment in middle-aged female rats with stroke. Stroke 38: 2150-2156, 2007.

31. Garbuzova-Davis S, Haller E, Tajiri N, Thomson A, Barretta J, Williams SN, Haim ED, Qin H, Frisina-Deyo A, Abraham JV, et al: Blood-spinal cord barrier alterations in subacute and chronic Stages of a rat model of focal cerebral ischemia. J Neuropathol Exp Neurol 75: 673-688, 2016.

32. Ma Y, Wang J, Wang Y and Yang GY: The biphasic function of microglia in ischemic stroke. Prog Neurobiol 157: 247-272, 2017.

33. Xu L, He D and Bai Y: Microglia-mediated inflammation and neurodegenerative disease. Mol Neurobiol 53: 6709-6715, 2016.

34. Benarroch EE: Microglia: Multiple roles in surveillance, circuit shaping, and response to injury. Neurology 81: 1079-1088, 2013.

35. Lee Y, Lee SR, Choi SS, Yeo HG, Chang KT and Lee HJ Therapeutically targeting neuroinflammation and microglia after acute ischemic stroke. Biomed Res Int 2014: 297241, 2014.

36. Kawabori M and Yenari MA: The role of the microglia in acute CNS injury. Metab Brain Dis 30: 381-392, 2015.

37. Nam KN, Woo BC, Moon SK, Park SU, Park JY, Hwang JW, Bae HS, Ko CN and Lee EH: Paeonol attenuates inflammation-mediated neurotoxicity and microglial activation. Neural Regen Res 8: 1637-1643, 2013.

38. Himaya SW, Ryu B, Qian ZJ and Kim SK: Paeonol from Hippocampus kuda Bleeler suppressed the neuro-inflammatory responses in vitro via NF-kB and MAPK signaling pathways. Toxicol In Vitro 26: 878-887, 2012.

This work is licensed under a Creative Commons Attribution-NonCommercial-NoDerivatives 4.0 International (CC BY-NC-ND 4.0) License. 